\title{
Cardiac troponin I for predicting right ventricular dysfunction and intermediate risk in patients with normotensive pulmonary embolism
}

\author{
K. Keller • J. Beule $\cdot$ A. Schulz $・$ M. Coldewey $\cdot$ \\ W. Dippold • J. O. Balzer
}

Published online: 12 December 2014

(C) The Author(s) 2014. This article is published with open access at Springerlink.com

\begin{abstract}
Background Right ventricular dysfunction (RVD) and cardiac troponin I (cTnI) are important tools for risk stratification in pulmonary embolism (PE). We investigate the association of RVD and cTnI in normotensive PE patients and calculate a cTnI cut-off level for predicting RVD and submassive PE. Methods Clinical, laboratory, radiological and echocardiagraphic data were analysed. Patients were categorised into groups with or without RVD and compared focussing on cTnI. Effectiveness of cTnI for predicting RVD and submassive PE was tested.

Results One hundred twenty-nine normotensive PE patients, 71 with and 58 without RVD, were included. Patients with RVD were older (75.0 years $(61.3 / 81.0)$ vs. 66.0 years $(57.7 /$ $75.1), P=0.019)$. cTnI $(0.06 \mathrm{ng} / \mathrm{ml}(0.02 / 0.23)$ vs. $0.01 \mathrm{ng} / \mathrm{ml}$ $(0.00 / 0.03), P<0.0001)$ and D-dimer values $(2.00 \mathrm{mg} / 1(1.08 /$
\end{abstract}

The study was conducted in St. Vincenz and Elisabeth Hospital Mainz (KKM).

K. Keller $\cdot$ A. Schulz $\cdot$ M. Coldewey

Department of Medicine II, University Medical Center Mainz

(Johannes Gutenberg-University Mainz), Langenbeckstr. 1,

55131 Mainz, Germany

K. Keller $(\bowtie) \cdot$ M. Coldewey

Center for Thrombosis and Haemostasis, University Medical Center Mainz (Johannes Gutenberg-University Mainz), Mainz, Germany

e-mail: karsten.keller@unimedizin-mainz.de

J. Beule $\cdot$ W. Dippold

Department of Internal Medicine, St. Vincenz and Elisabeth Hospital

Mainz (KKM), Mainz, Germany

J. O. Balzer

Department of Radiology and Nuclear Medicine, Catholic Clinic

Mainz (KKM), Mainz, Germany

J. O. Balzer

Department of Diagnostic and Interventional Radiology, University

Clinic, Johann Wolfgang Goethe-University Frankfurt/Main,

Frankfurt am Main, Germany
$4.05)$ vs. $1.23 \mathrm{mg} / \mathrm{l}(0.76 / 2.26), P=0.016)$ were higher in $\mathrm{PE}$ with RVD. cTnI was associated with RVD (OR 3.95; $95 \%$ CI $1.95-8.02, p=0.00014)$. AUC for cTnI diagnosing RVD was 0.79 , and for submassive PE0.87. Cut-off values for cTnI predicting RVD and submassive PE were $0.01 \mathrm{ng} / \mathrm{ml}$, with a negative predictive value of $73 \%$. cTnI was positively correlated with age, D-dimer and creatinine.

Conclusions In normotensive PE patients, $\mathrm{cTnI}$ is helpful for risk stratification and excluding RVD. cTnI elevation is correlated with increasing age and reduced kidney function.

Keywords Pulmonary embolism · Thrombosis · Venous thromboembolism $\cdot$ Cardiac troponin I $\cdot$ Tachycardia

\section{Introduction}

Acute pulmonary embolism (PE) is potentially lifethreatening [1-11]. Mortality from an acute PE event is closely related to the initial haemodynamic status and cardiac adaptation [1, 2, 4, 10, 12-17]. The diagnostic finding of right ventricular dysfunction (RVD) or positive cardiac troponin (cTn) levels in normotensive PE patients appears to alter the patient's prognosis significantly $[1-4,7-9,11-14,18-26]$.

We aimed to investigate the association of RVD and cardiac troponin I (cTnI) in normotensive PE patients and the potential cTnI for exclusion of RVD. Moreover, a further objective was to investigate parameters that have an impact on elevation of cTnI levels.

\section{Patients and methods}

We performed a retrospective analysis of normotensive patients with confirmed acute PE, who were treated in the Internal Medicine Department of the St. Vincenz and Elisabeth Hospital Mainz, Germany, between May 2006 and June 2011. 
Patients were identified by searching the hospital information system database (ICD diagnostic code of PE: I26).

\section{Enrolled subjects}

Patients were eligible for our analysis if they had a confirmed acute PE, were treated in the Internal Medicine Department of the hospital, were haemodynamically stable (systolic blood pressure $\geq 90 \mathrm{mmHg}$ ) according to the European Society of Cardiology (ESC) guidelines [4] and American Heart Association (AHA) scientific statement [21], an accurate transthoracic echocardiography (TTE) of acute phase was assessed and patients were at least 18 years of age. Diagnosis of acute PE was confirmed by an identified filling defect in the pulmonary artery system in a computed tomography pulmonary angiogram of the chest (CT) or positive venous ultrasound/phlebography of an extremity consistent with deep vein thrombosis (DVT) in patients with typical symptoms of PE (chest pain or dyspnoea) and a detected positive D-dimer or scintigraphic ventilationperfusion (V/Q) scan read as high probability for PE. If the diagnosis of $\mathrm{PE}$ was not confirmed or the criteria mentioned above were not fulfilled, patients were not included in this analysis. Studies with retrospective analysis of diagnostic standard data do not need an ethics statement in Germany.

\section{Analysed data}

Retrospectively analysed data comprised symptoms, medical history, physical examination, laboratory parameters (cTnI, creatine kinase (CK), creatinine, D-dimer), complications and examination results.

\section{Definitions}

Per definition, cTnI values were elevated if they exceeded $0.1 \mathrm{ng} / \mathrm{ml}$, and D-dimer values if they exceeded $0.110 \mathrm{mg} / \mathrm{l}$. RVD in TTE was defined according to the ESC guidelines [4] and AHA scientific statement [21] as abnormal motion of the interventricular septum, right ventricular (RV) hypokinesis, pressure overload with tricuspid valve insufficiency or RV enlargement (quotient of end-diastolic lateral-septal RV diameter/end-diastolicleft ventricular lateral-septal diameter $>0.9$, referring to AHA scientific statement [21]).

According to the ESC guidelines [4] and AHA scientific statement [21] the PE patients of our study with RVD or pathological cTnI levels were classified as submassive PE with an intermediate risk and those without both were classified as low-risk PE.
Fig. 1 Cardiac troponin I level in PE patients with and without RVD

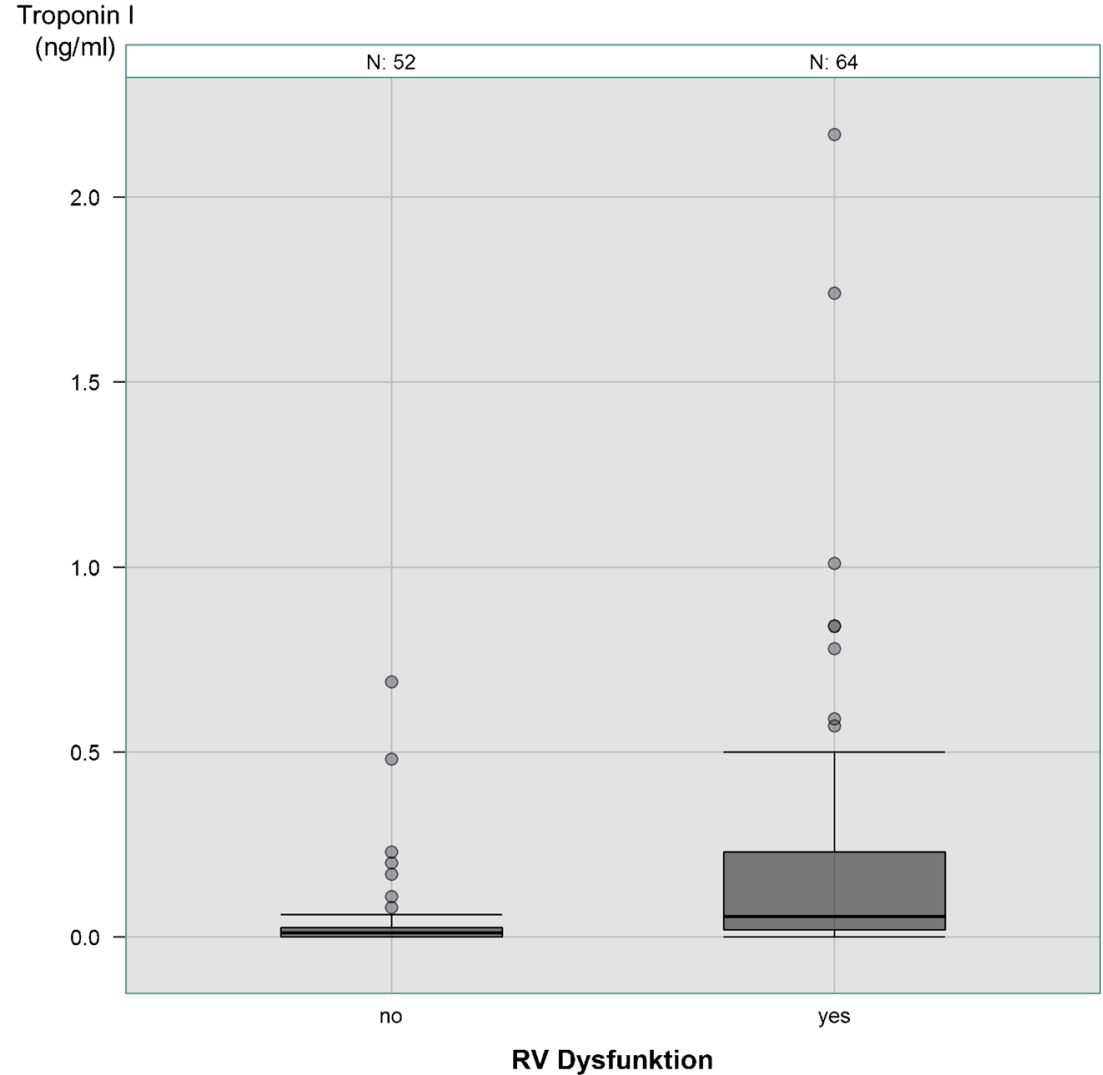




\section{Study groups}

According to TTE results the normotensive PE patients were included in one of the two study groups: the PE group with RVD or the PE group without RVD.

\section{Statistical analysis}

We compared the groups especially focussing on cTnI. Effectiveness of cTnI to predict RVD was tested. Continuous distributed variables were described by median and interquartile range and tested with the Mann-Whitney U-test if they were not normally distributed or by mean, standard deviation (SD) and tested with the Student $t$-test if distribution was normal. Discrete variables were compared with the Chisquare test. A value of $P<0.05$ was considered to be statistically significant.

Receiver operating characteristic (ROC) curve and Youden index were calculated to test the effectiveness of cTnI to predict RVD as well as cTnI to predict submassive PE. Multivariate regression analysis was performed to test coherence of cTnI, gender, age, CK, creatinine, and D-dimer on RVD. Spearman's rank correlation was calculated for age, cTnI, CK, creatinine, D-dimer, systolic blood pressure, diastolic blood pressure, heart rate and systolic pulmonary artery pressure (sPAP).

$\mathrm{R}$ version 2.14.1 from R Development Core Team (2011) (R Foundation for Statistical Computing, Vienna, Austria) was used for data processing.

Table 1 Characteristics of normotensive PE patients with and without RVD

\begin{tabular}{|c|c|c|c|}
\hline & PE without RVD $(n=58)$ & PE with RVD $(n=77)$ & $p$-value \\
\hline Sex (women) & $58.6 \%(34)$ & $60.6 \%(43)$ & 0.97 \\
\hline Age (years) & $66.0(57.7 / 75.1)$ & $75.0(61.3 / 81.0)$ & 0.019 \\
\hline \multicolumn{4}{|l|}{ Comorbidities } \\
\hline Surgery or trauma in last 3 months before PE event & $24.1 \%(14)$ & $14.1 \%(10)$ & 0.22 \\
\hline DVT or PE in patient's history & $24.6 \%(14)$ & $25.4 \%(18)$ & 0.92 \\
\hline DVT & $72.4 \%(42)$ & $69.0 \%(49)$ & 0.82 \\
\hline Lung infarction with pneumonia & $31.0 \%(18)$ & $52.1 \%(37)$ & 0.026 \\
\hline In-hospital death & $0 \%(0)$ & $1.4 \%(1)$ & 0.92 \\
\hline \multicolumn{4}{|l|}{ Symptoms } \\
\hline Chest pain & $34.5 \%(20)$ & $32.4 \%(23)$ & 0.95 \\
\hline Dyspnoea & $84.5 \%(49)$ & $85.9 \%(61)$ & 0.98 \\
\hline Haemoptysis & $3.4 \%(2)$ & $4.2 \%(3)$ & 0.82 \\
\hline Syncope or collapse & $5.2 \%(3)$ & $14.1 \%(10)$ & 0.17 \\
\hline Tachycardia & $20.7 \%(12)$ & $52.1 \%(37)$ & 0.00051 \\
\hline \multicolumn{4}{|l|}{ Physical examination } \\
\hline Systolic blood pressure (mmHg) & $151.4 \pm 23.5$ & $143.6 \pm 24.8$ & 0.070 \\
\hline Diastolic blood pressure (mmHg) & $80.9 \pm 20.4$ & $79.5 \pm 15.3$ & 0.65 \\
\hline Heart rate (beats/min) & $80.5(70.0 / 97.2)$ & $100.0(85.3 / 108.0)$ & $<0.0001$ \\
\hline \multicolumn{4}{|l|}{ ECG } \\
\hline Incomplete or complete RBBB & $3.5 \%(2)$ & $19.7 \%(14)$ & 0.013 \\
\hline S1Q3type & $3.5 \%(2)$ & $14.1 \%(10)$ & 0.083 \\
\hline \multicolumn{4}{|l|}{ Laboratory } \\
\hline Cardiac troponin I (ng/ml) & $0.01(0 / 0.03)$ & $0.06(0.02 / 0.23)$ & $<0.0001$ \\
\hline Creatine kinase (U/l) & $62.0(44.0 / 87.0)$ & $65.0(41.0 / 105.3)$ & 0.71 \\
\hline Creatinine (mg/dl) & $1.00(0.80 / 1.11)$ & $1.10(0.90 / 1.38)$ & 0.0062 \\
\hline D-dimer (mg/l) & $1.23(0.76 / 2.26)$ & $2.00(1.08 / 4.05)$ & 0.016 \\
\hline \multicolumn{4}{|l|}{ Echocardiography } \\
\hline Systolic PA pressure (mmHg) & $22.79 \pm 14.89$ & $43.00 \pm 16.06$ & $<0.0001$ \\
\hline Submassive PE stage (= existing RVD or elivated cTnI levels $(>0.1 \mathrm{ng} / \mathrm{ml})$ ) & $10.3 \%(6)$ & $100 \%(77)$ & $<0.0001$ \\
\hline
\end{tabular}

Continuous variables are described by median, 25th and 75th percentile, if they had a skewed distribution (|skewness $\mid>1)$. Nearly normally distributed variables are presented as mean values and standard deviation. Discrete variables are described through relative and absolute frequencies. Discrete variables were tested with the Chi-square test for contingency tables; continuous variables were analysed with Student's T Test if they were normally distributed the Mann-Whitney-U test on skewed distribution 


\section{Results}

Altogether, 182 patients with acute and confirmed PE were identified in the hospital information system database, but only 129 patients met the criteria of inclusion; 77 women and 52 men with normotensive PE were included in this analysis. The diagnosis of PE was confirmed in $84.4 \%$ by CT,10.1 \% by V/Q scan and $5.5 \%$ by DVT diagnosis in ultrasound/phlebography with typical symptoms of PE and positive D-dimer.

While $45.0 \%$ of the PE patients did not show RVD in TTE, $55.0 \%$ had RVD criteria. Patients with RVD were older (75.0 years $(61.3 / 81.0)$ vs. 66.0 years $(57.7 / 75.1), P=0.019)$, showed higher levels of heart rate $(100.0$ beats/min $(85.3 /$ $108.0)$ vs. 80.5 beats/min $(70.0 / 97.2), P<0.0001)$, sPAP $(43.00 \pm 16.06 \mathrm{mmHg}$ vs. $22.79 \pm 14.89 \mathrm{mmHg}, P<0.0001)$, cTnI (0.06 ng/ml (0.02/0.23) vs. $0.01 \mathrm{ng} / \mathrm{ml}(0.00 / 0.03)$, $P<0.0001)$ (Fig. 1), D-dimer (2.00 mg/l (1.08/4.05) vs. $1.23 \mathrm{mg} / \mathrm{l}(0.76 / 2.26), P=0.016)$ and creatinine $(1.10 \mathrm{mg} / \mathrm{dl}$ $(0.90 / 1.38)$ vs. $1.00 \mathrm{mg} / \mathrm{dl}(0.80 / 1.11), P=0.0062)$, more frequently had a lung infarction with pneumonia (52.1 vs. $31.0 \%, P=0.026)$ and an incomplete or complete right bundle branch block (RBBB) (19.7 vs. $3.5 \%, P=0.013$ ) (Table 1$)$. cTnI was not higher in PE patients $>65$ years than in those $\leq 65$ years $(0.03 \mathrm{ng} / \mathrm{ml}(0.01 / 0.16)$ vs. $0.02(0.00 / 0.08), P=$
0.10). In the PE group without RVD, $10.3 \%$ of patients showed positive cTnI values. Of the $129 \mathrm{PE}$ patients, 52 were classified as low-risk PE and 77 as submassive PE (Table 1).

Analysis of the ROC curve showed an AUC of 0.79 for cTnI predicting RVD with an optimal cut-off value of $0.01 \mathrm{ng} / \mathrm{ml}$. Of the normotensive PE patients, $58.6 \% \mathrm{had}$ cTnI values above $0.01 \mathrm{ng} / \mathrm{ml}$. Sensitivity was $80 \%(95 \%$ CI 68-89\%), specificity $67 \%$ (95\% CI 53-80\%), positive predictive value (PPV) $75 \%$ (95\% CI 63-85\%) and negative predictive value (NPV) $73 \%$ (95\% CI 58-85\%). AUC value and cTnI cut-off value predicting RVD as well as sensitivity, specificity, positive and negative predictive value have already been published in another context [24]. We have quoted these results once again to complete the general view on this topic.

Analysis of the ROC curve revealed an AUC of 0.87 for cTnI predicting submassive PE with an optimal cut-off value of $0.01 \mathrm{ng} / \mathrm{ml}$ (Fig. 2). Sensitivity was calculated as $81 \%$ (95\% CI 70-90\%), specificity 76\% (95\% CI 61-87\%), PPV $84 \%$ (95 \% CI 73-92\%) and NPV $73 \%$ (95 \% CI 58-85\%).

In multivariable logistic regression a strong association between cTnI and RVD was proven (OR 3.95; 95\% CI 1.95-8.02, $p=0.00014$ ) (Table 2). Spearman's rank correlation showed a positive correlation between cTnI and D-dimer $(r=0.33)$, sPAP $(r=0.32)$ and creatinine $(r=0.31)$, respectively (Fig. 3$)$.
Fig. 2 Receiver operating characteristic (ROC) curve with area under the curve (AUC) for effectiveness of cardiac troponin I to predict submassive PE with intermediate risk

\section{ROC-Plot for Troponin I}

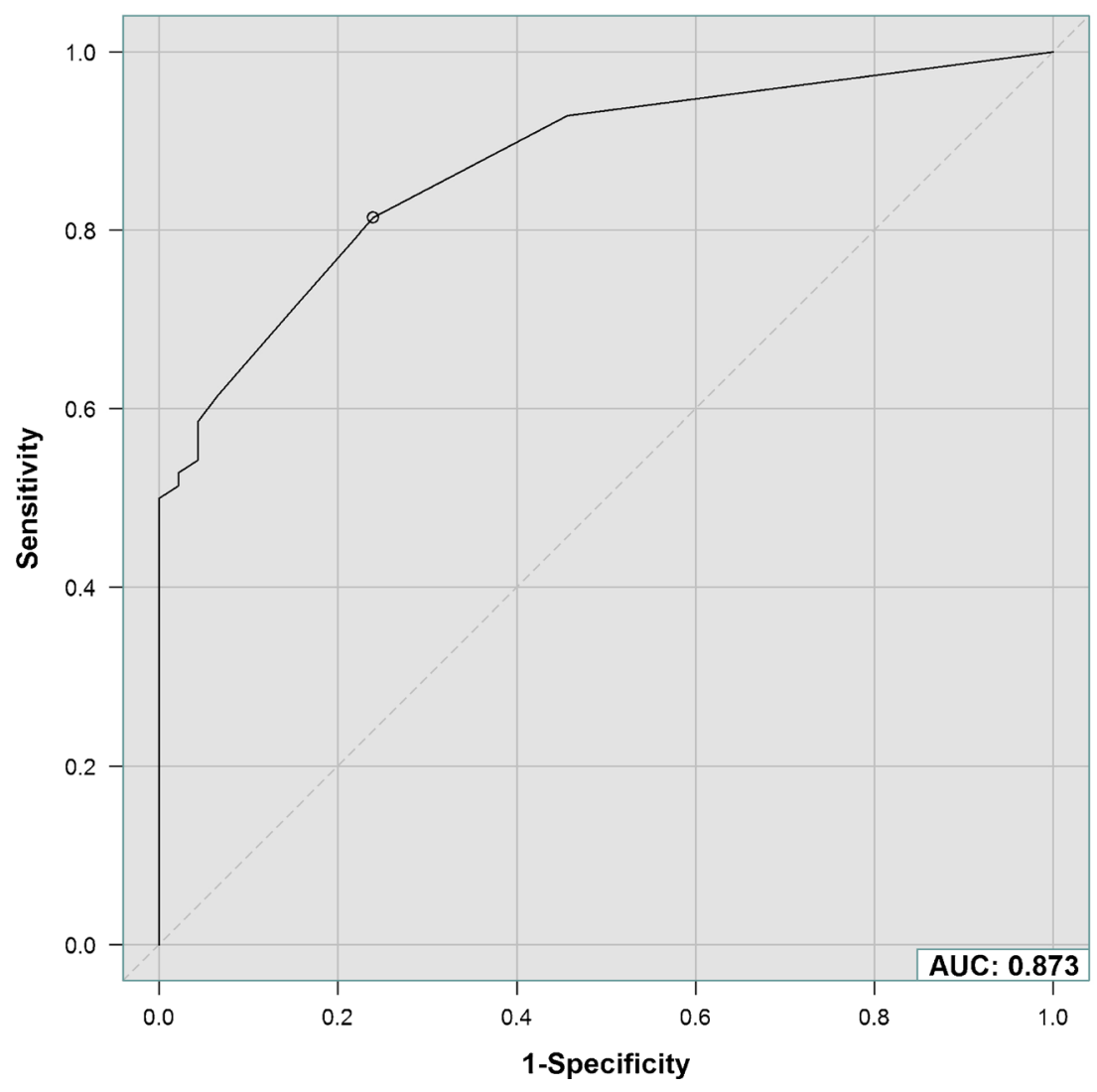


Table 2 Multivariate logistic regression to detect the coherence of $\mathrm{RVD}$ and $\mathrm{cTnI}$ and other parameters

\begin{tabular}{lll}
\hline & OR $(95 \% \mathrm{CI})$ & $p$-value \\
\hline Gender & $0.721(0.263-1.977)$ & 0.52 \\
Age & $1.199(0.715-2.011)$ & 0.49 \\
Log (cardiac troponin I) & $3.954(1.949-8.024)$ & $\mathbf{0 . 0 0 0 1 4}$ \\
CK & $1.843(0.287-11.822)$ & 0.52 \\
Creatinine & $1.331(0.721-2.457)$ & 0.36 \\
D-dimer & $0.964(0.588-1.581)$ & 0.88 \\
\hline
\end{tabular}

\section{Discussion}

Echocardiography, CT and cardiac biomarkers, particularly cTn, are important tools for risk stratification in normotensive PE patients. In acute PE, presence of RVD or elevated cTn levels is associated with increased mortality $[1,2,4,7,9,10$, 12-16, 18-22, 27-31].

Our study confirms a strong association between cTnI and RVD in normotensive PE patients. The calculated OR of 3.95 is in accordance with the literature [27, 32, 33]. AUC for cTnI predicting RVD was 0.79, which is similar to published AUC values of other studies [34, 35].

We calculated a cut-off value of $0.01 \mathrm{ng} / \mathrm{ml}$ for cTnI predicting RVD [24], which is in concordance with other study results [34]. Sensitivity, specificity, PPV and NPV were similar to other studies [34] and especially the PPV and NPV were high [24]. These results of AUC, cut-off value, sensitivity, specificity, PPV and NPV were previously published in an investigation for the comparison of three variations of RVD definitions [24]. But, in our opinion, these data are important to complete the general view on this topic and we have therefore quoted them once again.

Only $10.3 \%$ of the PE patients without RVD showed an elevated cTnI value. These patients had myocardial damage without detected RVD and therefore were also categorised as submassive PE with intermediate risk.

At a first glance, calculation of an AUC for cTnI predicting the submassive stage of $\mathrm{PE}$ seems to make no sense. But, after careful consideration this calculated AUC could be used to evaluate the importance of the parameter cTnI for assessment of submassive PE. Especially the small difference between the AUC of cTnI predicting RVD and
Fig. 3 Spearman's rank correlation coefficient for several parameters

\section{Spearmans rank correlation coefficients}

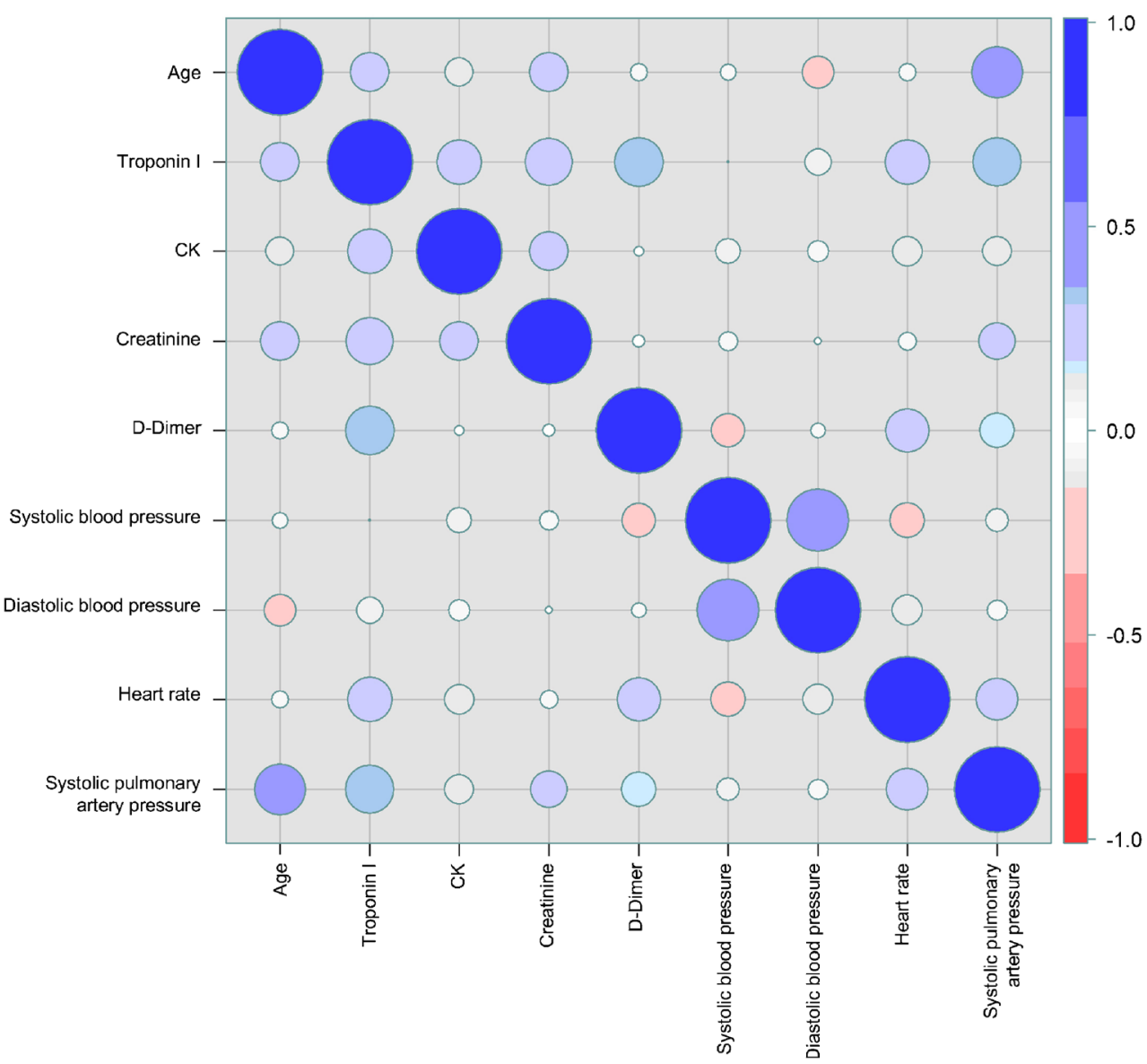


cTnI predicting submassive PE seems to indicate that in our analysis RVD was quite a lot more important for the classification of submassive PE than cTnI. The consistent cut-off values of cTnI for prediction of RVD and submassive PE indicate that the $\mathrm{cTnI}$ value to differentiate between normal and pathological is too high. A lower cut-off value for the definition of pathological findings and especially for defining the submassive stage of PE should be chosen in the future.

In a previous investigation, in $179 \mathrm{PE}$ patients of the identified 182 PE patients, we analysed the association of cTnI for risk stratification in haemodynamically unstable and normotensive PE patients together [36]. In the larger analysed group of PE patients, RVD diagnosis was made not only by TTE, but also by CT. In 3 of the 182 patients RVD was not detectable and these 3 patients were therefore not included in the analysis [36]. In these $179 \mathrm{PE}$ patients the association between $\mathrm{cTnI}$ and RVD was similar to the present analysis (OR 3.98) [36]. AUC for cTnI predicting RVD was 0.81 with a cut-off level of $0.01 \mathrm{ng} / \mathrm{ml}$ [36]. Increasing age, reduced kidney function and higher activation of coagulation system are connected with elevated cTnI values.

In all patients with an acute PE event, anticoagulation therapy is recommended with the aim to prevent early death and to avoid symptomatic recurrent VTE, especially fatal PE [31]. Thrombolytic treatment is not the standard treatment regime in normotensive PE patients, but could be considered if signs of haemodynamic decompensation appear [31]. In the 129 normotensive PE patients of our study we started anticoagulation treatment, but thrombolytic treatment was not given in any of these patients.

\section{Limitations and strength}

Limitations of this study are the small number of included patients and the retrospective character. With these data, we were not able to draw conclusions about follow-up outcome. Despite these limitations we were able to find answers to the main questions of the analysis.

\section{Conclusions}

In normotensive PE patients, $\mathrm{cTnI}$ is helpful for risk stratification and especially for exclusion of RVD. Increasing age, reduced kidney function and higher activation of coagulation system are connected with elevated cTnI values.

\section{Conflict of interest None.}

Funding None.

Open Access This article is distributed under the terms of the Creative Commons Attribution License which permits any use, distribution, and reproduction in any medium, provided the original author(s) and the source are credited.

\section{References}

1. Jimenez D, Uresandi F, Otero R, et al. Troponin-based risk stratification of patients with acute nonmassive pulmonary embolism: systematic review and metaanalysis. Chest. 2009;136(4):974-82.

2. Schellhaass A, Walther A, Konstantinides S, et al. The diagnosis and treatment of acute pulmonary embolism. Dtsch Arztebl Int. 2010;107(34-35):589-95.

3. Margato R, Carvalho S, Ribeiro H, et al. Cardiac troponin I levels in acute pulmonary embolism. Rev Port Cardiol. 2009;28(11):1213-22.

4. Torbicki A, Perrier A, Konstantinides S, et al. Guidelines on the diagnosis and management of acute pulmonary embolism: the Task Force for the Diagnosis and Management of Acute Pulmonary Embolism of the European Society of Cardiology (ESC). Eur Heart J. 2008;29(18):2276-315.

5. McRae S. Pulmonary embolism. Aust Fam Physician. 2010;39(7): $462-6$.

6. Konstantinides S, Goldhaber SZ. Pulmonary embolism: risk assessment and management. Eur Heart J. 2012;33:3014-22.

7. Kucher N, Rossi E, De Rosa M, et al. Prognostic role of echocardiography among patients with acute pulmonary embolism and a systolic arterial pressure of $90 \mathrm{~mm} \mathrm{Hg}$ or higher. Arch Intern Med. 2005;165(15):1777-81.

8. Becattini C, Vedovati MC, Agnelli G. Prognostic value of troponins in acute pulmonary embolism: a meta-analysis. Circulation. 2007;116(4):427-33.

9. Kreit JW. The impact of right ventricular dysfunction on the prognosis and therapy of normotensive patients with pulmonary embolism. Chest. 2004;125(4):1539-45.

10. Ohigashi H, Haraguchi G, Yoshikawa S, et al. Comparison of biomarkers for predicting disease severity and long-term respiratory prognosis in patients with acute pulmonary embolism. Int Heart J. 2010;51(6):416-20.

11. Masotti L, Righini M, Vuilleumier N, et al. Prognostic stratification of acute pulmonary embolism: focus on clinical aspects, imaging, and biomarkers. Vasc Health Risk Manag. 2009;5(4):567-75.

12. Goldhaber SZ. Assessing the prognosis of acute pulmonary embolism: tricks of the trade. Chest. 2008;133(2):334-6.

13. Giannitsis E, Muller-Bardorff M, Kurowski V, et al. Independent prognostic value of cardiac troponin $\mathrm{T}$ in patients with confirmed pulmonary embolism. Circulation. 2000;102(2):211-7.

14. Jimenez D, Diaz G, Molina J, et al. Troponin I and risk stratification of patients with acute nonmassive pulmonary embolism. Eur Respir J. 2008;31(4):847-53.

15. Sanchez O, Trinquart L, Caille V, et al. Prognostic factors for pulmonary embolism: the prep study, a prospective multicenter cohort study. Am J Respir Crit Care Med. 2010;181(2):168-73.

16. Haddad F, Doyle R, Murphy DJ, et al. Right ventricular function in cardiovascular disease, part II: pathophysiology, clinical importance, and management of right ventricular failure. Circulation. 2008;117(13):1717-31.

17. Kucher N, Wallmann D, Carone A, et al. Incremental prognostic value of troponin I and echocardiography in patients with acute pulmonary embolism. Eur Heart J. 2003;24(18):1651-6.

18. Fremont B, Pacouret G, Jacobi D, et al. Prognostic value of echocardiographic right/left ventricular end-diastolic diameter ratio in 
patients with acute pulmonary embolism: results from a monocenter registry of 1,416 patients. Chest. 2008;133(2):358-62.

19. Becattini C, Vedovati MC, Agnelli G. Right ventricle dysfunction in patients with pulmonary embolism. Intern Emerg Med. 2010;5(5): 453-5.

20. Grifoni S, Olivotto I, Cecchini P, et al. Short-term clinical outcome of patients with acute pulmonary embolism, normal blood pressure, and echocardiographic right ventricular dysfunction. Circulation. 2000;101(24):2817-22.

21. Jaff MR, McMurtry MS, Archer SL, et al. Management of massive and submassive pulmonary embolism, iliofemoral deep vein thrombosis, and chronic thromboembolic pulmonary hypertension: a scientific statement from the American Heart Association. Circulation. 2011;123(16):1788-830.

22. Kasper W, Konstantinides S, Geibel A, et al. Prognostic significance of right ventricular afterload stress detected by echocardiography in patients with clinically suspected pulmonary embolism. Heart. 1997;77(4):346-9.

23. Ribeiro A, Lindmarker P, Juhlin-Dannfelt A, et al. Echocardiography Doppler in pulmonary embolism: right ventricular dysfunction as a predictor of mortality rate. Am Heart J. 1997;134(3):479-87.

24. Keller K, Beule J, Schulz A, et al. Right ventricular dysfunction in hemodynamically stable patients with acute pulmonary embolism. Thromb Res. 2014;133:555-9.

25. Sanchez O, Trinquart L, Colombet I, et al. Prognostic value of right ventricular dysfunction in patients with haemodynamically stable pulmonary embolism: a systematic review. Eur Heart J. 2008;29(12):1569-77.

26. Mikulewicz M, Lewczuk J. Importance of cardiac biomarkers in risk stratification in acute pulmonary embolism. Cardiol J. 2008;15(1): $17-20$.

27. Ozsu S, Abul Y, Orem A, et al. Predictive value of troponins and simplified pulmonary embolism severity index in patients with normotensive pulmonary embolism. Multidiscip Respir Med. 2013;8(1): 34 .
28. Vieillard-Baron A, Page B, Augarde R, et al. Acute cor pulmonale in massive pulmonary embolism: incidence, echocardiographic pattern, clinical implications and recovery rate. Intensive Care Med. 2001;27(9):1481-6.

29. McConnell MV, Solomon SD, Rayan ME, et al. Regional right ventricular dysfunction detected by echocardiography in acute pulmonary embolism. Am J Cardiol. 1996;78(4):46973.

30. Palmieri V, Gallotta G, Rendina D, et al. Troponin I and right ventricular dysfunction for risk assessment in patients with nonmassive pulmonary embolism in the Emergency Department in combination with clinically based risk score. Intern Emerg Med. 2008;3(2):131-8.

31. Konstantinides S, Torbicki A, Agnelli G, et al. 2014 ESC guidelines on the diagnosis and management of acute pulmonary embolism: the Task Force for the Diagnosis and Management of Acute Pulmonary Embolism of the European Society of Cardiology (ESC) Endorsed by the European Respiratory Society (ERS). Eur Heart J. 2014.

32. Meyer T, Binder L, Hruska N, et al. Cardiac troponin I elevation in acute pulmonary embolism is associated with right ventricular dysfunction. J Am Coll Cardiol. 2000;36(5):1632-6.

33. Amorim S, Dias P, Rodrigues RA, et al. Troponin I as a marker of right ventricular dysfunction and severity of pulmonary embolism. Rev Port Cardiol. 2006;25(2):181-6.

34. Henzler T, Roeger S, Meyer M, et al. Pulmonary embolism: CT signs and cardiac biomarkers for predicting right ventricular dysfunction. Eur Respir J. 2012;39(4):919-26.

35. Logeart D, Lecuyer L, Thabut G, et al. Biomarker-based strategy for screening right ventricular dysfunction in patients with non-massive pulmonary embolism. Intensive Care Med. 2007;33(2):286-92.

36. Keller K, Beule J, Schulz A, et al. Troponin I as risk stratification marker in acute pulmonary artery embolism. Phlebologie. 2013;42: $261-9$. 